Didem Çelik'

İlker Ödemiș

Metin Korkmaz

Şükran Köse ${ }^{4}$

\section{A rare case of cardiac alveolar echinococcosis without any complications for eight years}

\author{
${ }^{1}$ Bakırçay University, Çiğli Education and Research Hospital, Department of Infectious Diseases and Clinical \\ Microbiology, Izmir, Turkey \\ ${ }^{2}$ Baskent University Adana Dr. Turgut Noyan Application and Research Center, Department of Infectious Diseases and \\ Clinical Microbiology, Adana, Turkey \\ ${ }^{3}$ Ege University, Department of Parasitology, Izmir, Turkey \\ ${ }^{4}$ University of Health Sciences, Izmir Tepecik Education and Research Hospital, Department of Infectious Diseases and \\ Clinical Microbiology, Izmir, Turkey
}

\section{Article history}

Received: 22 May 2021; Revision Requested: 16 June 2021; Revision Received: 8 August 2021; Accepted: 30 September 2021; Published: 26 November 2021

\section{Sir,}

Human alveolar echinococcosis (AE) is caused by the metacestode of Echinococcus multilocularis (EM) and it is considered to be the most potentially lethal parasitic zoonosis in the nontropical areas of the Northern Hemisphere [1]. AE is a rare parasitic disease and sporadic in Turkey. It can be encountered in dogs, cats, foxes, and domestic pigs which is transmitted via the fecal-oral route from contaminated foods and water or via close contact with infected animals. This disease is characterized by an infiltrative, tumor-like growth of the EM metacestode commonly in the liver of humans, followed by the lung, kidney, bone, and brain [2]. Cardiac involvement constitutes 0.5 to $2 \%$ of total cases and Cardiovascular surgery should be planned first [3]. The present study aims to discuss diagnosis, etiology, and treatment from a case with EM infection spread over the heart, lungs, and soft tissues.

A 26-year-old male patient with no history of chronic diseases presented to the outpatient clinic with swelling, enlargement, and pain in the left femoral region and difficulty in walking. Systemic inquiry of the patient revealed no additional complaints; personal history revealed smoking of 5 pack/year; the family history was unremarkable. On his physical examination, an increase was determined in the diameter of the proximal aspect of the left leg with a $3 \times 2 \mathrm{~cm}$ painful mass palpated on the lateral aspect of the left femoral region. Additional systemic examination was normal, vital signs were as following; body temperature: $36.5{ }^{\circ} \mathrm{C}$, pulse rate:78/min, blood pressure:110-70 mm/Hg. The patient, who had no history of trauma, underwent magnetic resonance imaging of the femur and pelvis. Regarding to the laboratory analyses, no abnormality was determined through full blood count, blood biochemistry, or autoimmune parameters. Radiological examination of the

\section{Correspondence:}

Didem Çelik

Department of Infectious Diseases and Clinical Microbiology, Bakırçay University, Çĭgli Education and Research Hospital, Yeni Mahalle, 8780/1. St. No:18, Çiğli, İzmir, 35620, Turkey Tel: +905543578646

E-mail: didemdestioglu@gmail.com patient demonstrated multiple space-occupying cystic lesions, the largest of them was $2 \times 5 \mathrm{~cm}$, in the left gluteus maximus, adductor, vastus lateralis and vastus medialis muscles of the quadriceps femoris group (Figure 1a-1b). Depending on the radiological examination EM infection was considered. When the patient's anamnesis was questioned again in terms of EM, it was learned that he was living with his dog and has a history of eating steak tartar a la turca, unwashed raw vegetables and fruits. The indirect hemagglutination test (IHA) test result for hydatid cyst was 1/1280 and the western blotting was positive (Figure 2). The brain and liver appeared to be normal on the whole-body magnetic resonance imaging, which was performed for screening purposes. A $27 \times 25 \mathrm{~mm}$ lesion with a slight thickness of hyperdense wall was detected in the posterior aspect of the cardiac interventricular septum (Figure 3) and multiple cystic lesions; the largest of which was $22 \times 17$ $\mathrm{mm}$, were detected in the bilateral lung parenchyma (Figure 4). Heterogeneous, hypodense lesions, which are $2 \mathrm{~cm}$ in diameter and attached to the surrounding tissues, were detected in the anterior and postero-lateral aspects of the left psoas muscle. PNM system for classification of human alveolar echinococcosis was PXN1M1 (Stage IV), therefore the cyst in the cardiac interventricular septum was consulted to the Department of Cardiology and Cardiovascular Surgery, however medical therapy was preferred as the patient did not consent for cardiac surgery as the surgery might be life-threatening. Albendazole was ordered at a dose of $2 \times 400 \mathrm{mg}$. The cyst compressing vena iliaca in the retroperitoneum was resected. In pathological examination of this cyst; a fibrous wall, $9 \times 5 \times 2.5 \mathrm{~cm}$ in size, containing adipose tissue areas on its outer surface, and a large number of cuticular membranes with the largest diameter of $4.5 \mathrm{~cm}$ and the smallest millimetric were observed. The cardiac magnetic resonance imaging performed after 3 months of medical therapy revealed regression in the size of the intracardiac cyst. On the 6th month and 1st-year control visits, no progression was determined in the size of cysts with no additional complaint. Furthermore no side effects were observed. 
1a

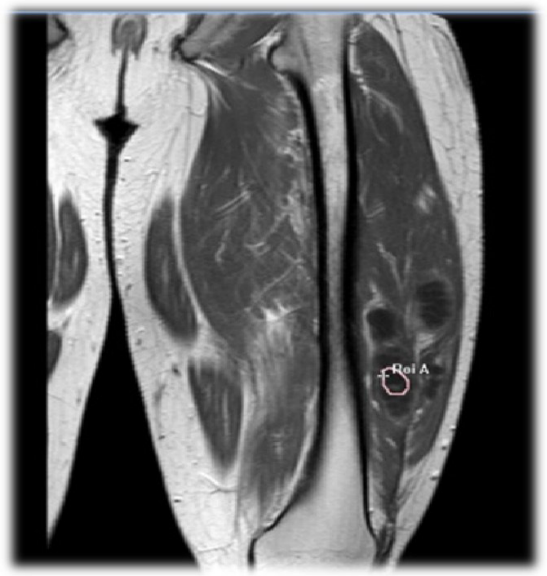

$1 \mathbf{b}$

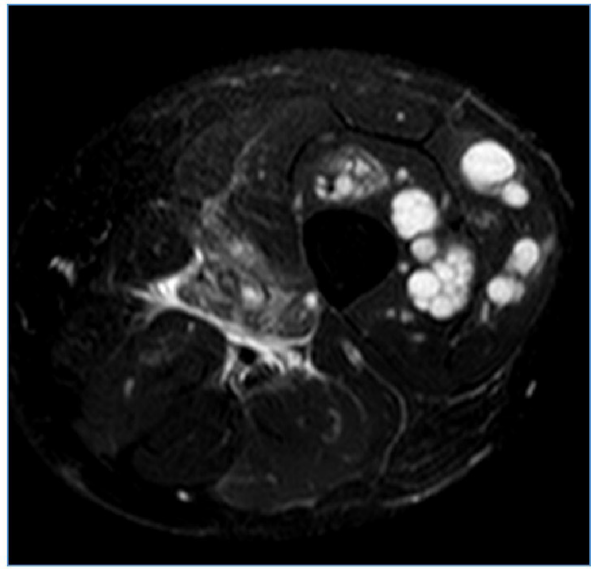

Figures 1 1a-1b. Multiple space-occupying cystic lesions, the largest of which was $2 \times 5 \mathrm{~cm}$, in the left muscles of the quadriceps femoris group

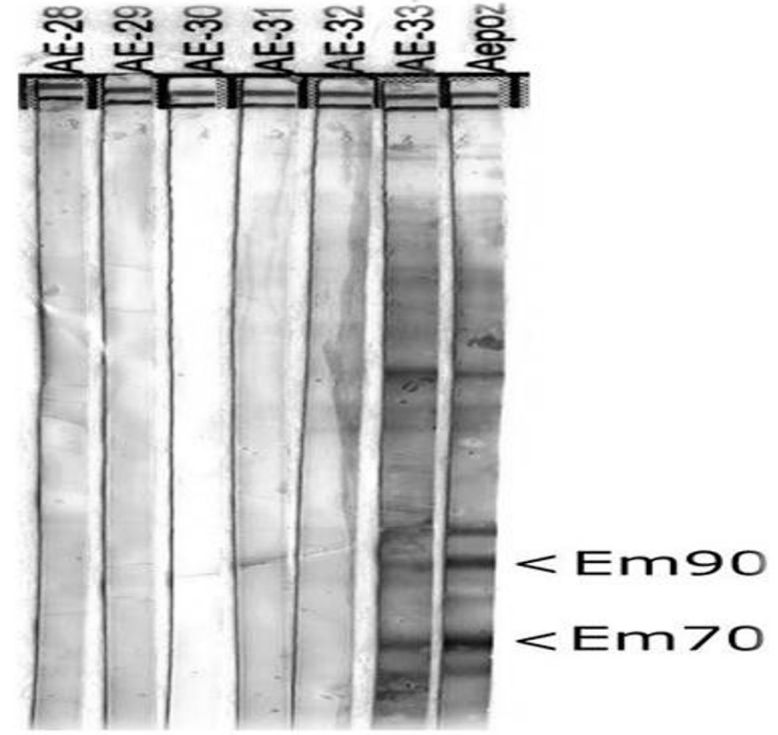

Figure 2 The presence of both 70- and 90-kDa bands (Em70 and Em90, respectively) was interpreted as a positive result for Alveolar echinococcosis (AE). Aepoz; positive control, AE-33; presented case, AE-28-32 negative cases for $A E$

After completing the first year, despite all risks the patient discontinued albendazole treatment voluntarily and continued to be followed up at close intervals. The patient has been followed up for eight years until now, he is still alive without any complications. During the follow-up period, whole-body imaging was repeated, the patient had no new cardiac complaints and no change was observed in the lesions.
EM is a zoonotic infection with multisystem involvement and a mortality rate of $4 \%$. AE is endemic especially in eastern Turkey. Of the AE cases, $87.4 \%$ reported in our country are from East and Southeast Anatolia [4]. The incubation period ranges from 5 to 15 years, the diagnosis usually delays because of the long incubation period $[5,6]$. In general, it involves primarily in the liver and then reaches to the pancreas, spleen, lungs, retroperitoneum, heart, brain, bone, and soft tissues via the extrahepatic spread. Lungs and brain involvement accounts for $20 \%$ of the spreads. The interesting point of our case was that the patient had a cyst in his heart despite no cysts in his liver, this reflects a very rare situation.

The diagnosis is made based on geographical prevalence, anamnesis, clinical findings, serological tests, and radiological findings. But in some cases, the diagnosis can be more difficult because the disease can present with various symptoms. The area where the patient lives, the foods he eats, and his occupation should be questioned. Computerized tomography and magnetic resonance imaging are helpful in diagnosis. Serology is more sensitive and specific than imaging [7]. IHA, Enzym Linked Immunosorbent Assay, and Indirect Fluorescent Antibody Test are the most useful and most commonly used serologic tests for diagnosis of $A E[7,8]$. In this case; the serologic diagnosis was done by IHA and western blot. The diagnostic specificity is $94.1 \%$, and the sensitivity is $100 \%$ in cases where IHA and WB tests are performed together [9]. Although in some cases, immune deficits may predispose to parasitic infections, in this case immune analyzes could not be performed because human leukocyte antigen typing and interleukin levels analysis were not available in our hospital. However, immunodeficiency was not considered in the patient who did not have a history of frequent infections and did not have anergic purified protein derivative (PPD) test.

In the differential diagnosis, metastatic tumors, bacterial metastatic abscesses, other parasitic infections, tuberculo- 


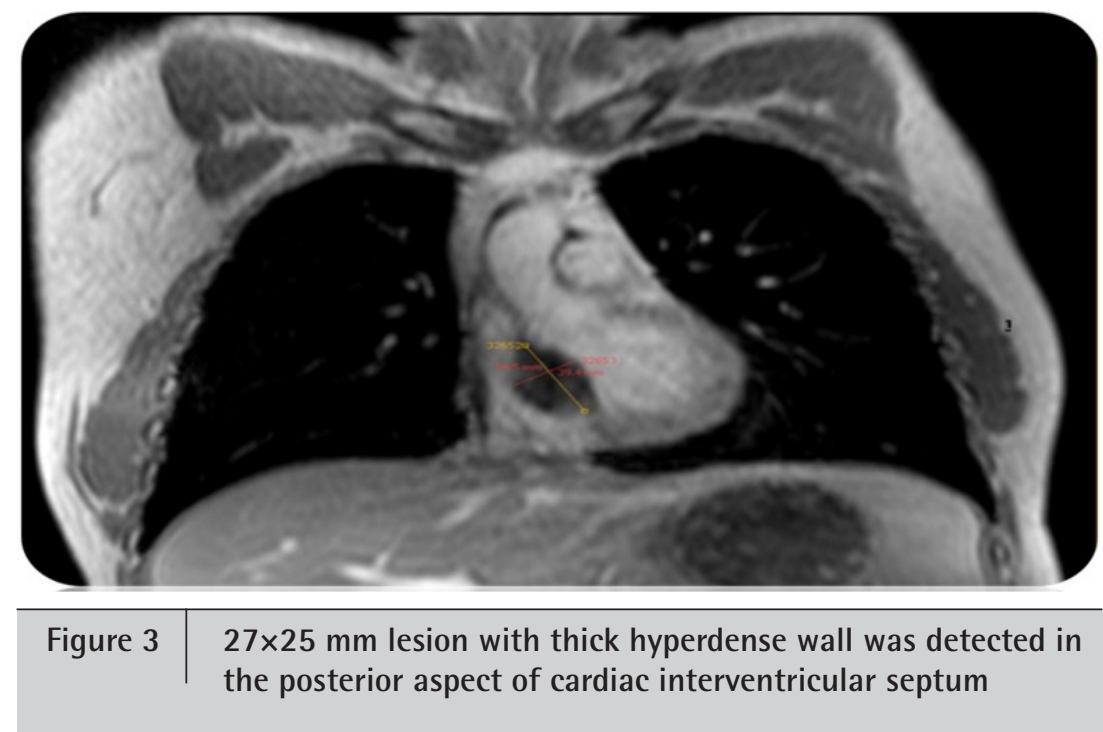

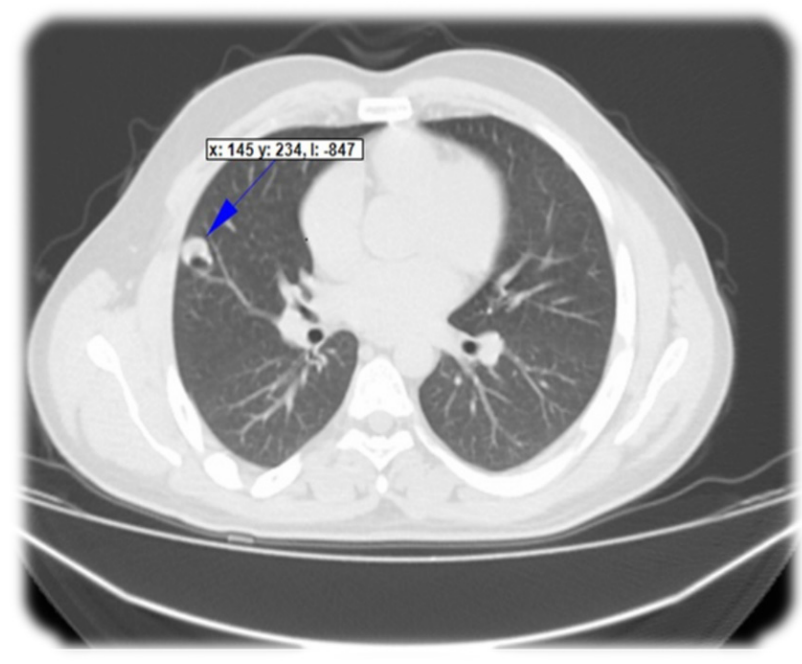

Figure $4 \quad$ Multiple cystic lesions in the bilateral lung parenchyma

sis should be considered. AE may mimic the tumor of the site where it is located especially if it's located in the liver. It can make distant organ metastasis by blood vessel invasion like tumors. Cardiac involvement constitutes 0.5 to $2 \%$ of the total cases. Surgery is the preferred treatment. Venous filters may be used to prevent the spread of the disease. When complete removal of cysts is possible, both the recurrence rate is low and the prognosis is good [3]. In our case, the patient did not give consent to cardiac surgery, so we decided to follow the patient by close intervals and preferred medical treatment.

Albendazole, a benzimidazole derivative, is indicated for inoperable patients with two or more organs or more than one cyst in the peritoneal region [3]. Lifelong treatment is often necessary to inhibit the development of parasites in patients who cannot benefit from radical surgery [6]. As a side effect, liver dysfunction, alopecia, neutropenia, and leukopenia can be seen $[4,6]$. We didn't detect any drug side effects in our patient. When the patients don't receive medical treatment, $70 \%$ of patients die within 5 years, and $90 \%$ within 10 years [2]. Chemotherapy should be continued for at least 2 years after surgery and patients should be monitored for at least 10 years [2]. When surgery is not appropriate, albendazole should be continued to prevent the progression of infection [10]. Although the general approach for treatment is surgery or lifelong medical treatment, in our case, long-term survival with one-year medical treatment can be a good guide for the success of medical treatment.

In conclusion, the patients diagnosed with AE should undergo whole-body screening including the lungs, heart, brain, pancreas, spleen bone, and soft tissue as early as possible. Surgical resection is the curative option for the treatment of disease; however, medical therapy is also an option where surgery is risky. The treatment should be planned with a multidisciplinary team that should include at least one infectious disease physician, radiologist, hepatology specialist, surgeon, with experience [6].

\section{ACKNOWLEGDEMENTS}

This study was presented as a poster presentation at International Symposium on Parasitic Zoonoses TMC Parasitology Working Group 16-20 November 2016 Titanic Deluxe Hotel Belek- Antalya.

\section{FUNDING}

None to declare 


\section{CONFLICTS OF INTEREST}

The authors declare no conflicts of interest.

\section{REFERENCES}

1. Korkmaz M, Inceboz T, Celebi F, Babaoglu A, Uner A. Use of two sensitive and specific immunoblot markers, Em70 and Em90, for diagnosis of alveolar echinococcosis. J Clin Microbiol. 2004;42(7):33502. doi: 10.1128/JCM.42.7.3350-3352.2004.

2. Pohnan R, Ryska M, Hytych V, Matej R, Hrabal P, Pudil J. Echinococcosis mimicking liver malignancy: A case report. Int I Surg Case Rep. 2017;36:55-8. doi: 10.1016/j.jijscr.2017.04.032

3. Brunetti E, Kern P, Vuitton DA. Expert consensus for the diagnosis and treatment of cystic and alveolar echinococcosis in humans. Acta trop. 2010;114(1):1-16. doi: 10.1016/j.actatropica.2009.11.001

4. Aras Y, Sabancı PA, Boyalı 0 , Aydoseli $A$, Güllüoğlu $M$, Bilgiç $M B$, et al. Kraniyal Metastazlı Alveolar Ekinokok: Olgu Sunumu ve Literatürün Gözden Geçirilmesi. Türk Nöroşir Derg. 2014;24(3):298-305.

5. Spahn S, Helmchen B, Zingg U. Alveolar echinococcosis of the right adrenal gland: a case report and review of the literature. J Med Case Rep. 2016;10(1):325. doi: 10.1186/s13256-016-1115-0

6. Nail LC, Reimundes ER, Galluzzo CW, Lebowitz D, Ibrahim YL, Lobrinus $J A$, et al. Disseminated alveolar echinococcosis resembling metastatic malignancy: a case report. J Med Case Rep. 2017;11(1):113. doi: 10.1186/s13256-017-1279-2

7. Aydın Y, Altuntaş $B$, Eroğlu A, Oğul H, Aydınlı B. Treatment approach for pulmonary alveolar echinococcosis. Turk J Thorac Cardiovasc Surg. 2017;25(2):223-9. doi: 10.5606/tgkdc.dergisi.2017.12486

8. Dülger AC, Küçükoğlu ME, Akdeniz H, Avcu S, Kemik Ö. Case report: Budd-Chiari syndrome and esophageal variceal bleeding due to alveolar echinococcosis. Turkiye Parazitol Derg. 2010;34(3):187-90. PMID: 20954122

9. Yılmaz GR, Babür C. Ekinokokkosis Tanısı. Turk Hij Den Biyol Derg. 2007:64(3):35-44.

10. Torgerson PR, Schweiger A, Deplazes P, Pohar M, Reichen J, Ammann RW, et al. Alveolar echinococcosis: from a deadly disease to a well-controlled infection. Relative survival and economic analysis in Switzerland over the last 35 years. J Hepatol. 2008;49(1):72-7. doi:10.1016/j.jhep.2008.03.023 